\title{
Genome-Wide Comparative Analysis of the $\beta$ - glucosidase Family in Five Rosaceae Species and their Potential Role on Lignification of Stone Cells in Chinese White Pear
}

\section{Han Wang}

School of Life Science, Anhui Agricultural University, Hefei, China

Yang Zhang

Anhui Agricultural University

Yu Zhao

Anhui Agricultural University

Wenlong Han

Anhui Agricultural University

Jinjin Lu

Anhui Agricultural University

Xi Cheng

Anhui Agricultural University

Guohui Li

Anhui Agricultural University

Qing Jin

Anhui Agricultural University

Yongping Cai ( $\nabla$ ypcaiah@163.com )

Anhui Agricultural University

Research article

Keywords: $\beta$-glucosidase, pear, systematic analysis, relative expression analysis, lignification, stone cell

Posted Date: July 29th, 2020

DOI: https://doi.org/10.21203/rs.3.rs-46971/v1

License: (c) (1) This work is licensed under a Creative Commons Attribution 4.0 International License.

Read Full License 


\section{Abstract}

Background: The $\beta$-glucosidase BGLU in the glycoside hydrolase family $1(\mathrm{GH} 1)$ is involved in the sugar metabolism of the plant and plays an important role in maintaining the normal physiological function of the plant. Recent studies had shown that $\beta$-glucosidase was involved in plant lignification. The lignification in pear fruit is closely related to the formation of pear stone cells, but the $B G L U$ genes family has not been identified in pears.

Result: A total of 343 BGLU genes were identified from five species of Rosaceae (Pyrus bretschneideri, Prunus mume, Malus domestica, Prunus avium, Fragaria vesca). According to phylogenetic analysis, 50 PbBGLUs were divided into 8 groups. 298 syntenic pairs were found in intra- and inter-species collinear analysis of five Rosaceae species, found that pears and apples had more syntenic pairs than pear and the other three Rosaceae species. The Ka/Ks analysis of duplication PbBGLU genes in pear indicated that the main mode of expansion of the PbBGLUs was segmental replication and was mainly affected by purification. qRT-PCR showed that the three gene expression patterns of PbBGLU1, PbBGLU15 and PbBGLU16 were basically consistent with the change trend of pear fruit lignin and stone cell content, and may be involved in lignification and stone cell development of pear fruit. Subcellular localization showed that these three candidate genes were all located on the cell wall.

Conclusion: In this study, a genome-wide analysis of $B G L U$ genes in five Rosaceae species was carried out, and three candidate genes related to lignification and stone cell development of pear fruits were identified, which laid the foundation for a deeper understanding of the function of $B G L U$ genes in pear fruits and potential in changing pear fruit quality.

\section{Background}

In plants, Glycoside Hydrolase (GH) family $1 \beta$-glycosidases are believed to play important roles in many diverse processes including chemical defense against herbivory, lignification, hydrolysis of cell wallderived oligosaccharides during germination, and control of active phytohormone levels [1]. In recent years, more and more species of the $\beta$-glucosidase family had been discovered, and the function of the $\beta$ glucosidase gene had been more clearly understood. A total of 47 BGLUs were identified in Arabidopsis, of which AtBGLU1-6 was used for the accumulation of flavonoids in Arabidopsis [1, 2], AtBGLU9 and AtBGLU10 catalyzed the final step of anthocyanin formation in Arabidopsis [3]. AtBGLU15 displayed the highest catalytic efficiency for Q3G7R and K3G7R yielding their respective 7-0-rhamnosides as products [4]. AtBGLU18 and AtBGLU33 regulate ABA responses by increasing ABA levels through the hydrolysis of glucose-conjugated ABA (ABA-GE) [5, 6]. AtBGLU21-23 was related to the production of scopolin in Arabidopsis roots [7, 8]. The PEN2 gene (At2g44490, AtBGLU26) of Arabidopsis thaliana had been shown to encode a myrosinase-like activity which is limited to indolic glucosinolate substrate [9]. AtBGLU34-39 function as myrosinases for chemical defense against herbivores and pathogen attacks [10, 11]. $\beta$ glucosidase AtBGLU42 was a MYB72-dependent key regulator of rhizobacteria-induced systemic resistance and modulates iron deficiency responses in Arabidopsis roots [12]. There was also a lot of 
evidence that the $\beta$-glucosidase was involved in plant lignification. Back in 1994, coniferin-specific $\beta$ glucosidase was identified in xylem of two Pinus specie $[13,14]$. A study found in 1999 that Coniferin $\beta-$ glucosidase catalyzed the hydrolysis of monolignol glucosides to release the cinnamyl alcohols for oxidative polymerization to lignin [15]. At1g61810 (AtBGLU45), At1g61820 (AtBGLU46) and At4g21760 (AtBGLU47) in Arabidopsis play an important role in the synthesis of Arabidopsis lignin [16, 17]. Os4BGLU14, Os4BGLU16 and Os4BGLU18 found in the rice $\beta$-glucosidase family have been proved to be functional genes in rice lignin synthesis [18].In addition, a $\beta$-glucosidase was identified from the poplar tree and named 'PtrBGLU6', which plays an important role in poplar lignification and cell wall formation [19].

Hydrolysis of glycosides plays an important role in the metabolism of plants such as lignification [20]. Lignin is a phenylpropane polymer composed of three kinds of monolignols ( $p$-coumaryl alcohol, coniferyl alcohol and sinapyl alcohol) connected by different chemical bonds [21, 22]. These three monolignols are catalyzed by UDP-glucosyltransferase (UGT) into the monolignol glucosides ( $p$ coumarin, coniferin, syringin) [23,24], glycosylation will increase the solubility and stability of monolignols, which is beneficial to the transportation and storage of monolignols. Studies have found that a large amount of monolignol glucosides may be stored in the vacuoles of different xylem cells [25, 26].The research on the transport mechanism of monolignols is not very thorough, but the academic community currently believes that there are three transport mechanisms for the extracellular secretion of monolignols, namely passive diffusion (PD), vesicle-related exocytosis, and the use of $A B C$ transport factors or proton coupling to reverse transport Active transportation of ATP-consuming [21, 27, 28].Then these monolignol glucosides are transported to specific parts of the cell wall and hydrolyzed to lignin monolignols under the action of $\beta$-glucosidase, finally formed into guaiacyl (G), syringyl (S) and phydroxyphenyl $(\mathrm{H})$ units by laccase and peroxidase(Dicotyledons only have guaiacyl $(G)$ and syringyl (S) lignin) [29](Fig. 1).

Pear belongs to Rosaceae family and is one of the most important deciduous fruit trees in the world. It is widely cultivated in many countries [30]. "Dangshan Su" pear (Pyrus bretschneideri cv. Dangshan Su) is a diploid pear variety that originally from Dangshan County, Anhui Province, China which is not only has good flavor, high nutritional value, but also has medicinal value, and is very popular among people. However, due to its high content of stone cells, its economic value is not high enough [31]. Stone cells are a kind of peculiar cell in pear fruit, it is one of the important factors that affect pear fruit processing and fresh food quality. Current research shows that the size, number and density of stone cells have significant effects on pear fruit quality. The greater the diameter density of the cell mass, the more the stone cell content of pear fruit, the worse the fruit quality [32]. Stone cells are formed by secondary thickening of parenchyma cells, and lignin is one of the main components of stone cells [33, 34]. Lignin is a secondary metabolite, during pear fruit development, lignin synthesis, transport and deposition are closely related to stone cell development $[35,36]$.To this end, this study aims to conduct in-depth research on the pear $B G L U$ genes family using bioinformatics methods on the basis of previous studies, clarify the molecular evolution characteristics of the BGLU family, and screen out $\beta$-glucosidase gene related to the 
development of pear stone cells and lignification. With a view to providing a theoretical basis for the formation and regulation of the "Dangshan Su" pear stone cells.

\section{Result}

\section{Identification of BGLU genes in five Rosaceae species}

The BGLU family conserved domain (Pfam:PF00232) was used as the target query sequence, and the corresponding hidden Markov model was obtained from the Pfam website (http://pfam.xfam.org/) were used to identify the BGLU members. SMART (http://smart.embl-heidelberg.de/) was used to check whether there are characteristic domains, finally removed those redundant and repeated sequences. As a result, in total, we identified 343 BGLU genes from 5 Rosaceae species (Pyrus bretschneideri 50, Prunus mume 52, Malus domestica 132, Prunus avium 78, Fragaria vesca 31). The detailed information on the

gene ID, gene name, chromosome location, protein structure and the characteristics of the corresponding BGLU proteins were listed in Additional file 1: Table S1.

\section{Phylogenetic conserved motif and Exon-Intron Structures analyses of BGLU genes in Chinese white pear}

For all PbBGLUgenes, The phylogenetic tree was constructed with MEGA 7.0 software using the neighbor-joining $(\mathrm{NJ})$ (bootstrap = 1,000), and then divide them into 8 groups $(\mathrm{I}, \mathrm{II}, \mathrm{III}, \mathrm{IV}-\mathrm{A}, \mathrm{IV}-\mathrm{B}, \mathrm{IV}-\mathrm{C}, \mathrm{V}-\mathrm{A}, \mathrm{V}$ B)with supported bootstrap values(Fig. 2),We used the MEME online software to analyze the amino acid sequence of $P b B G L U$ genes, and identified 20 Motifs in all sequences (Additional file 1:Table S2, Fig. 2), All the PbBGLUs contain Motif1 (CTGGBSATEPYJVAHHQLLAHAAAVKLYREKYQA), only two genes do not contain Motif9 (WFEPASESKEDKAAALRALDF), Speculate that Motif1 and Motif9 are conserved gene motifs of PbBGLUs family. Only group I and group II contain Motif19, the other groups do not, and all members of group IV-C do not contain Motif2. It is speculated that these Motifs may have special significance. Previous studies implied that gene structural diversity can lead to the evolution of multigene families [37]. To better characterize and understand the structural diversity of the PbBGLUs, gene exon-intron analysis was carried out $\mathrm{Fig}$. 2囚, except that PbBGLU23 and PbBGLU24 have only one exon, the rest contain 7 or more exons, of which PbBGLU33 contains the most exons (21) and most gene exons range between 10-15. These results indicating the possible occurrence of alternative splicing during gene expression, which in turn leads to functional differences between these closely related PbBGLUs [37].

In order to more clearly understand the evolutionary relationship and related functions of $P b B G L U$ s, the phylogenic tree, including BGLUs from Arabidopsis thaliana, Poplar $[1,19]$ and pear was constructed (Fig. 3). We have marked the position of the 8 groups in the pear on this phylogenic tree and found that group I contains only PbBGLUs but not AtBGLUs and PtrBGLUs, indicating that some changes occurred among $B G L U$ s of different species during the evolutionary process [38]. PbBGLUs Group II were clustered together with Poplar PtrBGLU9 and PtrBGLU11, however the functions of these two genes are unclear. Arabidopsis AtBGLU12-17 was related to the use of Arabidopsis flavonoids, AtBGLU15 can catalyze the hydrolysis of Q3G7R, K3G7R and flavonol 3-0-glucosides [4], these genes and PbBGLU19 (group III) were 
clustered together, and it is speculated that PbBGLU19 may be involved in the hydrolysis of flavonoid glycosides in pear. PbBGLUs IV-A was clustered together with $A t B G L U(40,41,43,44)$ and some poplar $B G L U$ genes, but the functions of these genes have not yet been explored. The functions of AtBGLU45, AtBGLU46, AtBGLU47 in Arabidopsis and PtrBGLU6 in Poplar had been clarified, they can catalyze the hydrolysis of monolignol glucosides during lignification [16,17,19], the three genes PbBGLU1, PbBGLU15 and $P b B G L U 16$ in the PbBGLUs group IV-B were clustered together with these functional genes, speculating that $P b B G L U 1, P b B G L U 15$ and $P b B G L U 16$ may be able to affect the lignification by catalyze the hydrolysis of monolignol glucosides in pear fruit, which in turn affects the development of pear stone cells. PbBGLUs Group IV-C clustered together with AtBGLU42, AtBGLU42 is a myb72-dependent key regulator of rhizobacteria-induced systemic resistance and modulates iron deficiency responses in Arabidopsis roots [12]. AtBGLU1-6, which plays a role in the accumulation of flavonoids in Arabidopsis [2], was clustered together with PbBGLUs group V-A, speculating that $P b B G L U 28$ and $P b B G L U 29$ may have similar functions in pears. PbBGLUs group V-B clustered together with Arabidopsis AtBGLU34-39, AtBGLU34-39 function as myrosinases for chemical defense against herbivores and pathogen attacks. $[10,11]$

\section{Chromosome location and collinearity analysis of BGLUs family members of five Rosaceae species}

To further reveal how the $B G L U$ genes family evolved, we mapped the $B G L U$ genes to their respective chromosomes (Additional file 2: Fig. S1), 40 PbBGLUs (10 genes are not located on any chromosome) were unevenly distributed on 10 chromosomes (a total of 17 chromosomes of pear),Chromosome 7,10,13 had only one chromosome distribution, chromosome 11 had the most chromosome distribution (11). 40 PmBGLUs (12 unlocated) in Prunus mume were located on 8 chromosomes, except for only one gene was located on chromosomes 4 and 6, the other PmBGLU genes were relatively evenly located on the rest chromosome. Apple 114 (9 unlocated) MDBGLUs were located on 17 chromosomes, among which chromosome 3 and chromosome 11 are more distributed. The 42 PaBGLU(36 unlocated) genes of Prunus avium were located on 8 chromosomes, chromosome 3, 7, 8 had less PaBGLUs distributed, the remaining chromosomes had more PaBGLUs distributed and relatively uniform. The 31 FVBGLUs of strawberry were located on 7 chromosomes and were unevenly distributed. Among them, FvBGLUs on chromosomes 3 and 5 were concentrated located and the others chromosomes were only located a small amount.

Species homologous genes are divided into orthologous and paralogous, orthologous genes refer to the genes that exist in the ancestral genome and are subsequently transferred to different species because of species differentiation, these genes often have a high degree of similarity in structure and function! Paralogous genes refer to homologous genes in the same genome due to gene duplication, because homologous genes generated by replication do not have the same selection pressure effect, some mutations are prone to occur, which may lead to functional mutations [39].298 syntenic pairs were found in intra- and inter-species collinear analysis of five Rosaceae species (Fig. 4, Additional file 1:Table S 3),A total of 12 syntenic pairs were found between pears, among which PbBGLU3/PbBGLU7 gene pairs were on the same chromosome, PbBGLU7 and PbBGLU50 were collinear with PbBGLU3, PbBGLU19 and 
PbBGLU25 were collinear with PbBGLU33, PbBGLU44 and PbBGLU13 were collinear with PbBGLU22, these genes may be paralogous and may formed by the whole genome replication of pears a million years ago. Pear and apple have more syntenic pairs (41) than pear and plum (19), Pear and strawberry (15), Pear and cherry (11), these gene pairs between pear and other Rosaceae species may be the orthologous caused by the differentiation of species in the ancestral genome. Both pears and apples contain 17 chromosomes, and the number of chromosomes is double that of strawberries, cherries, and Mei. Previous studies have shown that the genome-wide replication (GWD) may be occurred greater than 50 million years ago, resulting in Pear and Apple chromosomes from 9 ancestral chromosomes to 17 chromosomes. Data from a three-way sequence alignment between predicted gene space in apple $(\sim$ $84 \mathrm{Mb})$ and experimentally derived EST data from pear ( $14.9 \mathrm{Mb})$, predicted gene spaces of apple and pear were compared, a value of $96.35 \%$ nucleotide identity was calculated between these two species of the tribe Pyreae. When the frequency of transitions and transversions was considered, the ratio $\mathrm{R}$ (transitions/transversions) was similar for apple-specific and pear-specific mutations [40], these may be the reasons why pears and apples have more syntenic pairs.

\section{PbBGLUgenes replication event analysis}

To determine the selection pressure in duplication of $P b B G L U$ s genes, the non-synonymous (Ka)/synonymous (Ks) values were calculated for the 12 gene pairs (Additional file 1: Table S4). The pear divergence time between these gene pairs was calculated with the period varied from 0.27-154.25 million years (Mya). It is generally believed that synonymous mutations are not subject to natural selection, while non-synonymous mutations are subject to natural selection. If $\mathrm{Ka} / \mathrm{Ks}>1$, it is considered to have a positive selection effect. If $\mathrm{Ka} / \mathrm{Ks}=1$, then there is a neutral selection. If $\mathrm{Ka} / \mathrm{Ks}<1$, it is considered to have purification selection effect. We calculated $\mathrm{Ka} / \mathrm{Ks}$ values of 12 gene pairs, only one group of genes PbBGLU3/PbBGLU7 Ka/Ks > 1, the replication mode is tandem, and the remaining 11 pairs of genomes $\mathrm{Ka} / \mathrm{Ks}<1$, the replication mode is segmental. The result suggested that the evolution of the BGLU gene pairs in the pear may be mainly due to fragment replication under purification selection and with limited functional divergence after the duplication events.

We performed sliding window analysis on these 12 gene pairs(Additional file 3: Fig. S2), It was found that $P b B G L U 3 / P b B G L U 7$ had a large number of $\mathrm{Ka} / \mathrm{Ks}$ ratios of coding sites more than 1 , experience strong positive choices during the evolution, while PbBGLU12/PbBGLU42, PbBGLU25/PbBGLU33, PbBGLU33/PbBGLU19, PbBGLU44/PbBGLU22 and PbBGLU49/PbBGLU20 had a small amount of $\mathrm{Ka} / \mathrm{Ks}$ ratios of coding sites more than 1 , suggested that these gene pairs may also experience some positive choices During the evolution.

\section{Promoter Regions of PbBGLUGenes}

The promoter sequence of 2000 bp upstream of the members of the PbBGLUs family was obtained from pear GigaDB database PLACE database, and then the promoter region was analyzed using the online software PlantCARE (http://bioinformatics.psb.ugent.be/webtools/plantcare/html/) Cis-acting element [41].The results shown that (Fig. 5, Additional file 1: TableS5): A total of 9 regulatory element including 
MBS, MRE, AC, LTR, HSE, TC-rich repeat, ABRE, ERE, CGTCA motifs were identified. Among them, the MBS element related to drought stress is the most, only 8 PbBGLU genes do not contain this element. Minimum number of AC elements, only 4 PbBGLU genes contain AC elements. Most genes contain light response element MRE, low temperature stress response element $L T R$, heat shock stress response element HSE, and defense response element TC-rich repeat. In addition, cis-acting elements related to hormone response (ABA response element ABRE, Ethylen response element ERE, and MeJR response element CGTCA motif) were also found in many genes of PbBGLU. These results suggested that in addition to its normal function, $P b B G L U$ s may also participate in light response, stress response, plant self-defense response and hormone response process.

\section{Expression characteristics of Chinese white pear BGLU genes}

We had built the phylogenic tree, including $B G L U$ s from Arabidopsis thaliana, Poplar $[1,19]$ and pear, and found out that PbBGLU1, PbBGLU15 and PbBGLU16 in the PbBGLUs IV-B of pears were clustered together with these functional genes AtBGLU45, AtBGLU46, AtBGLU47 and PtrBGLU6 which can hydrolysis monolignol glucosides during Lignification $[16,17,19]$. In order to verify whether PbBGLU1, PbBGLU15 and PbBGLU16 have similar functions. We selected all PbBGLU genes in IV-B, and also selected all $P b B G L U$ genes in IV-B, IV-C, V-A, V-B which were relatively close to IV-B on the phylogenic tree (a total of 25 PbBGLUs) to do qRT-PCR analysis. The purpose was to explore these genes in different tissue parts of pears (leaves, buds, stems, flowers) and pear fruit at different developmental stages $(15,39,47,55,63,79,145$ days after flowering) expression profiles (Fig. 6). It was found that PbBGLU36 and PbBGLU42 were expressed neither in different tissue parts nor in different developmental stages of fruit. The expression levels of the other 23 PbBGLUs were generally higher in leaves, buds, flowers and young fruits $(15,39,47,55$ days after flowering), this result is consistent with previous research that the $\beta$ glucosidase can be found mainly in young vegetative parts $[42,43]$. Compared with other genes, PbBGLU38 and PbBGLU43 are more uniformly expressed in all tissue parts and all stages, and may perform a certain indispensable function in pear fruit trees. The expression levels of PbBGLU28 and PbBGLU29 gene in flowers, 79 DAF (when the lignification stop in fruit) $[31,35,36]$ and 145 DAF (when the fruit was fully mature) $[31,35,36]$ relatively high. Moreover, these two genes were clustered together with AtBGLU1-6 which related to the accumulation of flavonoids in Arabidopsis [2], so PbBGLU28 and $P b B G L U 29$ may participate in the accumulation of flavonoids in pear. The expression levels of the three genes PbBGLU1, PbBGLU15 and PbBGLU16 have similar characteristics, they are relatively high in leaves and flowers, and relatively low in buds and stems, all three genes were expressed at different stages of fruit development, and its expression shows a trend of rising first and then falling, with similar trend of the content of lignin and stone cells in "Dangshan Su" pear fruit[35, 36], and the three genes had similar expression patterns with the key genes involved in regulation of the lignin synthesis pathway [44]. These results suggested that the three genes PbBGLU1, PbBGLU15, and PbBGLU16 in PbBGLUs IV-B may be functional genes involved in the hydrolysis of monolignol glucosides in the pear lignin synthesis pathway.

\section{Subcellular Localization of PbBGLU1, PbBGLU15 and PbBGLU16}

Page $7 / 24$ 
As early as 1995 , it was found that $P$. contorta $\beta$-glucosidase activity was located in the cell wall of secondary xylem tissue [14]. AtBGLU45 and AtBGLU46 of Arabidopsis thaliana were also located in the cell wall [16].The rice Os4BGlu16 tagged with a C-terminal GFP was transiently expressed in tobacco leaf epithelial cells and found that the GFP signal was exclusively localized to the plasma membrane and extracellular space in both intact and plasmolysed cells, consistent with an apoplastic or cell wall localization [18]. In this study, in order to explore the PbBGLU1, PbBGLU15, PbBGLU16 subcellular localization, PbBGLU-GFP expression vectors were constructed and transformed into N. tabacum. As shown in Fig. 7, green fluorescence signals from the expressed fusion PbBGLU1-GFP, PbBGLU15-GFP and PbBGLU16-GFP genes were specifically distributed in the cell wall.

\section{Discussion}

The $B G L U$ genes family only identified in a few species. We had identified a total of $343 B G L U$ genes from five species of Rosaceae, of which $50 B G L U$ genes were identified from pears. The number was similar to that of Arabidopsis 47 [1] and Poplar 48 [19], More than 31 rice [45] and 26 maize [46]. In the analysis and identification of the rice $\mathrm{GH} 1$ family, most of the genes appear to have been derived from duplications that occurred after the divergence of rice and Arabidopsis thaliana lineages from their common ancestor, and the two plants share only 8 common gene lineages [45]. Maize identified 26 BGLU genes (Zmbg/u1-Zmbglu26), Since there were no two Zmbg/u paralogs that had identical molecular properties, people concluded that gene subfunctionalization in maize occurs much more rapidly than gene duplication [46]. In this study, we constructed a phylogenetic tree of PbBGLUs, and then divided into 8 groups. Conserved motif analysis of PbBGLUs found that all the PbBGLUs contain Motif1, 48 PbBGLUs contain motif9, speculated that the two motifs may be related to catalytic activity. To adapt different new functions that are suitable for changes in the environment, gene structure was commonly diversified during the evolution of multigene families [47], for Arabidopsis BGLU genes family 13 exon-12 intron organization was considered as the ancestral gene structure, and the pattern with 13 exons was the most common $[1,37]$. In this study, we had analyzed exon-intron Structures of $P b B G L U$ s and the results suggested that PbBGLU23 and PbBGLU24 contain only one exon, PbBGLU33 contains the most exons (21) and most gene exons range between $10-15$, the result suggested that compared with AtBGLUs, PbBGLUs may have some genetic structure diversity.

About 50 million years ago, genome-wide replication (GWD) led to the transformation of pears and apples from 9 to 17 chromosomes, gene spaces of apple and pear were compared, a value of $96.35 \%$ nucleotide identity was calculated between these two species of the tribe Pyreae. When the frequency of transitions and transversions was considered, the ratio $\mathrm{R}$ (transitions/transversions) was similar for apple-specific and pear-specific mutations [40]. These previous research results explain well why the more syntenic pairs between pear and apple. To further reveal the potential mechanism of evolution of the $B G L U$ genes family, both the segmental and tandem duplication events were analyzed in pear, the result shown that only one gene pair was tandem replication, and the remaining 11 pairs of genomes replication mode was segmental. In addition, previous studies reported that tandem duplication helped to generate new genes and often occurred in the large and rapidly evolving gene family, such as NBS-LRR gene family [48], 
whereas, segmental duplication usually occurred in the slowly evolving gene family, such as MYB and $B B X$ gene family $[38,49]$.

Previous studies found that $B G L U$ genes is not only related to lignin synthesis, but also play important roles in many diverse processes including chemical defense against herbivory, hydrolysis of cell wallderived oligosaccharides during germination, and control of active phytohormone levels [1]. In this study, the analysis of the PbBGLUs promoter found that most genes contain not only the light-responsive ciselement MRE, the low-temperature stress response element LTR, the heat shock stress response element HSE and the defense response cis element TC-rich repeat but also the hormone response-related ciselement ( $A B A$ response element ABRE, Ethylen response cis-element ERE, and MeJR response ciselement CCGCA motif).

Phylogenetic tree containing pears, Arabidopsis and poplar showed that pear's three genes PbBGLU1, $P b B G L U 15$ and PbBGLU16 in the PbBGLUs IV-B group were clustered together with these genes AtBGLU45, AtBGLU46, AtBGLU47 and PtrBGLU6 which can hydrolysis monolignol glucosides during Lignification $[16,17,19]$. Then qRT-PCR results suggested that the three genes PbBGLU1, PbBGLU15, and PbBGLU16 may be functional genes involved in the hydrolysis of monolignol glucosides in the pear lignin synthesis pathway. Previous studies had shown that the role of pear monolignol glucosides were actually necessary for transmembrane transport $[19,21]$, the monolignol glucosides form can increase the solubility and stability of monolignols, which is very beneficial for the transportation of lignin, then to specific locations at the cell wall, lignin begins to deglycosylate and polymerize [29]. Many previous studies had reported that genes were localized to the cell wall and extracellular $[14,16,18]$. In this study we found that green fluorescence signals from the expressed fusion PbBGLU1-GFP, PbBGLU15-GFP and PbBGLU16-GFP genes were specifically distributed in the cell wall, consistent with previous research.

\section{Conclusion}

In this study, a total of $343 B G L U$ genes were identified from five Rosaceae species. The phylogenetic tree divided 50 PbBGLUs into 8 groups (I, II, III, IV-A, IV-B, IV-C, VA, VB), Collinearity between five Rosaceae species found that there are more collinear gene pairs between pears and apples. The $\mathrm{Ka} / \mathrm{Ks}$ ratio analysis of duplication genes in pear indicated that the main mode of expansion of pear BGLU genes family is segmental replication and is mainly affected by purification. qRT-PCR showed that the three genes expression patterns of PbBGLU1, PbBGLU15 and PbBGLU16 were basically consistent with the change trend of pear fruit lignin and stone cell content, and may be involved in stone cell development and lignification of pear fruit. Subcellular localization showed that these three candidate genes were all located on the cell wall.

\section{Materials And Methods}

\section{Identification of BGLU genes in five Rosaceae species}


The Chinese white pear genome database was obtained from the Pear Genome Project (http://gigadb.org/dataset/10008). The genome databases of two Rosaceae plants (Fragaria vesca, Prunus avium) were downloaded from genome database for Rosaceae (GDR) (https://www.rosaceae.org/). The genome sequence data of Prunus mume was downloaded from the Prunus mume genome project (http://gigadb.org/dataset/10008).

The sequences of apple (Malus domestica) was downloaded from Joint Genome Institude (http://www.jgi.doe.gov/).

To identify putative $B G L U$ genes from five Rosaceae plants, several approaches were employed. Using a BGLU family characteristic domain (Pfam: PF00232) as a query sequence in accordance with the HMM configuration file (PF00232) of BGLU, we searched for candidate genes using the HMM with E-values < 1e - 10. We downloaded the HMM profile (PF00232) from the Pfam website (https://pfam.xfam.org/)[50]. The online site SMART (http://smart.embl-heidel-berg.de/) [51] was used to determine the domain PF00232 of BGLU. The online analysis tool EXPASY (http://web.expasy.org/compute_pi/) was used to predict and analyze the physical and chemical properties of the obtained BGLU amino acid sequence to understand the sequence properties and characteristics of the amino acids of BGLU family members.

\section{Phylogenetic conserved motif and Exon-Intron Structures analyses of BGLU genes in pear}

Sequence alignment of PbBGLUs proteins was done using the ClustalW tool in MEGA 7.0 software. The phylogenetic tree was constructed with MEGA 7.0 software using the neighbor-joining ( $N J$ ) (bootstrap = 1,000) [52]. The conservative motifs was analyzed by MEME (http://meme.sdsc.edu/meme4_3_0/intro.html) software the maximum value of the motif was set to 20, and the motif length was set between 6 and 200[53]. Gene Structure Server (http://gsds.cbi.pku.edu.cn) was used to generate exon-intron map [54].

Phylogenetic analysis of BGLUs from Arabidopsis, Pyrus bretschneideri and Populus. The software MEGA 7.0 was used to construct the phylogenetic tree. The amino-acid sequences of Arabidopsis and Populus were obtained from phytozome (https://phytozome.jgi.doe.gov/pz/portal.html\#).

\section{Chromosome location and collinearity analysis of BGLU family members of five Rosaceae species}

Obtain the location information of $B G L U$ on the chromosome from the genome database, and use Maplnspect (http://mapinspect.software.informer.com/) software to complete the location map of $B G L U$ on the chromosome.

Multiple Collinearity Scan toolkit (MCSscanX) [55] and BLASTP (1e-10, identity $>80 \%$ ) was used to determine the Gene duplication and microsynteny among these five species Chinese pear (Pyrus bretschneideri), Mei (Prunus mume), apple (Malus domestica), Sweet cherry (Prunus avium) and Strawberry (Fragaria vesca). collinear graph was draw by Super Circos.

\section{PbBGLU genes replication event analysis}


non-synonymous substitution rate( $\mathrm{Ka}) \square$ synonymous substitution rate $(\mathrm{Ks})$, and $\mathrm{Ka} / \mathrm{Ks}$ ratio was calculated by DnaSP v5.0, Ka/Ks ratio of each duplicate gene pairs was determined to calculate the selection pressure and sliding window analysis was carried out to analyze the Synonymous (Ks) and nonsynonymous (Ka) rates of $P b B G L U$ genes encoding sites paralogs. The Ks values were used to estimate the approximate date of every duplicated event occurred in pear, seeing the formula: $T=K s / 2 \lambda \times 10-6$ Mya $(\lambda=6.5 \times 10-9)[56]$.

\section{Analysis of cis-Acting Elements in the Promoter Regions of PbBGLUs}

To analyze the promoter regions of the $P b B G L U$ genes, 2,000 bp regions upstream of these genes were examined based on the positions of the genes provided in pear GigaDB database PLACE database [41] was employed to examine putative cis-acting regulatory DNA elements in the promoter regions of these genes.

\section{qRT-PCR analysis}

The materials were from 'Dangshan Su' pear (Pyrus bretschneideri cv. Dangshan Su) with the same growing environment for 40 years in Dangshan County, Anhui Province, China. We collected the mature leaves, buds, stem segments and flowers on March 30, and collected the fruit on April 15th (15 day after flowering), May 14th (39DAF), and May 22th (47DAF), June 4th (55DAF), June 12th (63DAF), June 28th (79DAF), August 30th (145DAF). Each sample is frozen at -80 degrees liquid nitrogen and brought back to the laboratory for use.

The TIANGEN RNAprep pure (Tiangen, Beijing, China) was used to extract the total RNA according to the manufacturer's instructions. Reverse transcription used EasyScript One-Step gDNA Removal and cDNA Synthesis SuperMix kit (TransGen Biotech, Beijing, China). The Beacon Designer 7 software was used to design and check the gene-specific primers (Table S6). The pear tubulin gene (forward primer: $5^{\prime}$ AGAACAAGAACTCGTCCTAC-3'; reverse peimer: 5'-GAACTGCTCGCTCACTCTCC-3') was used as reference gene [57] The reaction system is: $10 \mu \mathrm{L}$ of SYBR® Premix Ex TaqTM II (2) (TaKaRa), PbBGLUs upstream primer $0.8 \mu \mathrm{L}, \mathrm{PbBGLUs}$ downstream primer $0.8 \mu \mathrm{L}$ and $1 \mu \mathrm{L}$ cDNA. The PCR reaction procedure was: predenaturation at $95^{\circ} \mathrm{C}$ for $30 \mathrm{~s}$; denaturation at $95^{\circ} \mathrm{C}$ for $5 \mathrm{~s}$, annealing at $60^{\circ} \mathrm{C}$ for $20 \mathrm{~s}$, extension at $72^{\circ} \mathrm{C}$ for $10 \mathrm{~min}, 40$ cycles. Each sample was subjected to 3 biological replicates, and the relative expression levels of the $P b B G L U$ genes were calculated using the $2^{-\triangle \triangle C T}$ method [58].

\section{Subcellular localization of PbBGLU1, PbBGLU15 and PbBGLU16}

The PbBGLU1, PbBGLU15 and PbBGLU16 full CDS were cloned based on genomic information and combine these 3 genes with pCambia1304 and pCambia1301 vectors (Clontech, Beijing, country-region China) which have both CaMV35S promoter and GFP gene. Information on the primers, vectors and restriction sites required for the subcellular localization of the PbBGLU1, PbBGLU15 and PbBGLU16 were shown in Table S7. After electroporation of these construction into Agrobacterium tumefaciens EHA105, using pCAMBIA1304 vector as negative control. The infection solution was injected into the epidermis of 
Nicotiana tabacum leaves, after culturing in the dark for 3 days, the glass slide was made and placed under the FV1200 laser confocal microscope (Olympus Corporation, Japan) to observe the distribution of fusion protein.

\section{Abbreviations}

BGLU: $\beta$-glucosidase; GH1: glycoside hydrolase family 1; HMM: Hidden Markov Model; Mya: Million years ago; NJ: neighbor-joining; qRT-PCR: Real-Time PCR; ABC transporter: ATP-bindingcassette transporter.

\section{Declarations}

\section{Acknowledgments}

The authors would like to thank everyone who contributed to this article.

\section{Funding}

This study was supported by National Natural Science Foundation of China (No.31640068),

Anhui Agricultural University 2020 Graduate Innovation Foundation (2020ysj-50),

China Postdoctoral Science Foundation (No. 2019M662135),

Anhui Provincial Natural Science Foundation (No. 2008085QC100),

Anhui Provincial Postdoctoral Science Foundation (No. 2019B319),

Scientific Research Foundation of Anhui Agricultural University (No. 2019zd01 and yj2019-17),

Medjaden Academy \& Research Foundation for Young Scientists (No. MJR20201119),

Anhui University Student Innovation Training Project (201910364162).

\section{Availability of data and materials}

The Chinese white pear genome database was obtained from the Pear Genome Project (http://gigadb.org/dataset/10008). The genome databases of two Rosaceae plants (Fragaria vesca, Prunus avium) were downloaded from genome database for Rosaceae (GDR)

(https://www.rosaceae.org/). The genome sequence data of Prunus mume was downloaded from the Prunus mume genome project (http://gigadb.org/dataset/10008). The sequences of apple (Malus domestica) was downloaded from Joint Genome Institude (http://www.jgi.doe.gov/). Arabidopsis and Populus sequences were obtained from phytozome (https://phytozome.jgi.doe.gov/pz/portal.html\#).

\section{Authors' contributions}


YCai conceived and designed the experiments, HWang, YZhang, YZhao performed the experiments, WLHan, JJLu, XCheng, GHLi analyzed the data, HWang and YZhang wrote the paper. All authors reviewed and approved the final submission.

\section{Ethics approval and consent to participate}

The experiments did not involve endangered or protected species. No specific permits were required for these locations/activities because the pears used in this study were obtained from a horticultural field in Dangshan County, Anhui Province, China, which were demonstration orchards at Auhui Agricultural University.

\section{Consent for publication}

Not applicable.

\section{Competing interests}

The authors declare that they have no competing interests.

\section{References}

1. Xu Z, Luis L. Zeng LH, Mallikarjun L, David R, Cheng CL, Che M, Shih JEP and Asim E. Functional genomic analysis of Arabidopsis thaliana glycoside hydrolase family 1[J]. Plant Molecular Biology. 2004;55(3):343-367.

2. Ishihara H, Tohge T, Viehover P, Fernie AR, Weisshaar B, Stracke R. Natural variation in flavonol accumulation in Arabidopsis is determined by the flavonol glucosyltransferase BGLU6. J Exp Bot. 2016;67(5):1505-1517.

3. Miyahara T, Sakiyama R, Ozeki Y, Sasaki N. Acyl-glucose-dependent glucosyltransferase catalyzes the final step of anthocyanin formation in Arabidopsis. J Plant Physiol. 2013;170(6):619-624.

4. Roepke J, Bozzo GG. Arabidopsis thaliana beta-glucosidase BGLU15 attacks flavonol 3-0-betaglucoside-7-0-alpha-rhamnosides. Phytochemistry .2015;109:14-24.

5. Lee KH, Piao HL, Kim HY, Choi SM, Jiang F, Hartung W, Hwang I, Kwak JM, Lee IJ, Hwang I. Activation of glucosidase via stress-induced polymerization rapidly increases active pools of abscisic acid. Cell .2006;126(6):1109-1120.

6. Xu ZY, Lee KH, Dong T, Jeong JC, Jin JB, Kanno Y, Kim DH, Kim SY, Seo M, Bressan RA et al. A vacuolar beta-glucosidase homolog that possesses glucose-conjugated abscisic acid hydrolyzing activity plays an important role in osmotic stress responses in Arabidopsis. Plant Cell. 2012;24(5):2184-2199.

7. Hino F, Okazaki M, Miura Y. Effect of 2,4-dichlorophenoxyacetic acid on glucosylation of scopoletin to scopolin in tobacco tissue culture. Plant Physiol. 1982; 69:810-813. 
8. Ahn YO, Shimizu B, Sakata K, Gantulga D, Zhou C, Bevan DR, Esen A. Scopolin-hydrolyzing betaglucosidases in roots of Arabidopsis. Plant Cell Physiol. 2010;51(1):132-143.

9. Zhou C, Tokuhisa JG, Bevan DR, Esen A. Properties of beta-thioglucoside hydrolases (TGG1 and TGG2) from leaves of Arabidopsis thaliana. Plant Sci. 2012;191-192:82-92.

10. Barth C, Jander G. Arabidopsis myrosinases tgg 1 and tgg2 have redundant function in glucosinolate breakdown and insect defense. Plant J. 2006; 46:549-562.

11. Wittstock U, Burow M. Glucosinolate breakdown in arabidopsis: Mechanism, regulation and biological significance. Arabidopsis Book. 2010;8: e0134.

12. Zamioudis C, Hanson J, Pieterse CM. $\beta$-Glucosidase BGLU42 is a MYB72-dependent key regulator of rhizobacteria-induced systemic resistance and modulates iron deficiency responses in Arabidopsis roots. New Phytol. 2014;204(2):368-379.

13. Leinhos V, Udagama-Randeniya $P$ V, Savidge R A. Purification of an acidic coniferin-hydrolysing betaglucosidase from developing xylem of Pinus banksiana[J]. Phytochemistry. 1994;37(2).

14. Dharmawardhana D P, Carlson E J E. A p-Clucosidase from Lodgepole Pine Xylem Specific for the Lignin Precursor Coniferin[J]. Plant physiology. 1995;107(2):331-339.

15. Dharmawardhana D P, Ellis B E, Carlson J E. cDNA cloning and heterologous expression of coniferin B-glucosidase[J]. Plant Molecular Biology. 1999;40(2): 365-372.

16. Chapelle A, Morreel K, Vanholme R, Le-Bris P, Morin H, Lapierre C, Boerjan W, Jouanin L, DemontCaulet N. Impact of the absence of stem-specific beta-glucosidases on lignin and monolignols. Plant Physiol. 2012;160(3):1204-1217.

17. Escamilla-Trevino LL, Chen W, Card ML, Shih MC, Cheng CL, Poulton JE. Arabidopsis thaliana betaGlucosidases BGLU45 and BGLU46 hydrolyse monolignol glucosides. Phytochemistry. 2006;67(15):1651-1660.

18. Baiya S, Mahong B, Lee SK, Jeon JS, Ketudat Cairns JR. Demonstration of monolignol betaglucosidase activity of rice Os4BGlu14, Os4BGlu16 and Os4BGlu18 in Arabidopsis thaliana bglu45 mutant. Plant Physiol Biochem. 2018; 127:223-230.

19. Tsuyama T, Takabe K. Coniferin $\beta$-glucosidase is ionically bound to cell wall in differentiating xylem of poplar. Journal of Wood Science. 2015;61(4):438-444.

20. Julien L R, Brigitte H, Anne C, Simon H, Godfrey N. Glycosylation Is a Major Regulator of Phenylpropanoid Availability and Biological Activity in Plants[J]. Frontiers in Plant Science. 2016;7:735.

21. Barros J, Serk H, Granlund I, Pesquet E. The cell biology of lignification in higher plants. Ann Bot. 2015;115(7):1053-1074.

22. Boerjan W, Ralph J, Baucher M. Lignin Biosynthesis[J]. Annual review of plant biology. 2003;54(1):519-546.

23. Cheng X, Muhammad A, Li G, Zhang J, Cheng J, Qiu J, Jiang T, Jin Q, Cai Y, Lin Y: Family-1 UDP glycosyltransferases in pear (Pyrus bretschneideri): Molecular identification, phylogenomic 
characterization and expression profiling during stone cell formation. Mol Biol Rep. 2019;46(2):21532175.

24. Perkins M, Smith RA, Samuels L: The transport of monomers during lignification in plants: anything goes but how? Curr Opin Biotechnol. 2019; 56:69-74.

25. Steeves V, Förster H, Pommer U, Savidge R. Coniferyl alcohol metabolism in conifers - I. Glucosidic turnover of cinnamyl aldehydes by UDPG: coniferyl alcohol glucosyltransferase from pine cambium. Phytochemistry. 2001;57(7):1085-1093.

26. Tsuji Y, Fukushima K. Behavior of monolignol glucosides in angiosperms.J Agric Food Chem. 2004;52, 7651-7659.

27. Alejandro S, Lee Y, Tohge T, Sudre D, Osorio S, Park J, Bovet L, Lee Y, Geldner N, Fernie AR et al. AtABCG29 is a monolignol transporter involved in lignin biosynthesis. Curr Biol .2012;22(13):12071212.

28. Miao YC, Liu CJ. ATP-binding cassette-like transporters are involved in the transport of lignin precursors across plasma and vacuolar membranes. Proc Natl Acad Sci U S A .2010;107(52):2272822733.

29. Liu CJ. Deciphering the enigma of lignification: precursor transport, oxidation, and the topochemistry of lignin assembly. Mol Plant. 2012;5(2):304-317.

30. Wu J, Wang Z, Shi Z, Zhang S, Ming R, Zhu S, Khan MA, Tao S, Korban SS, Wang H, et al. The genome of the pear (Pyrus bretschneideri Rehd.). Genome Research .2012;23(2):396-408.

31. Yan C, Yin M, Zhang N, Jin Q, Fang Z, Lin Y, Cai Y. Stone cell distribution and lignin structure in various pear varieties. Scientia Horticulturae .2014;174:142-150.

32. Zhang J, Cheng X, Jin Q, Su X, Li M, Yan C, Jiao X, Li D, Lin Y, Cai Y. Comparison of the transcriptomic analysis between two Chinese white pears (Pyrus bretschneideri Rehd.) genotypes of different stone cells contents. PLoS One .2017;12(10): e0187114.

33. Su X, Zhao Y, Wang H, Li G, Cheng X, Jin Q, Cai Y. Transcriptomic analysis of early fruit development in Chinese white pear (Pyrus bretschneideri Rehd.) and functional identification of PbCCR1 in lignin biosynthesis. BMC Plant Biol .2019;19(1):417.

34. Cheng X, Li G, Manzoor MA, Wang H, Abdullah M, Su X, Zhang J, Jiang T, Jin Q, Cai Y et al: In Silico Genome-Wide Analysis of Respiratory Burst Oxidase Homolog (RBOH) Family Genes in Five FruitProducing Trees, and Potential Functional Analysis on Lignification of Stone Cells in Chinese White Pear. Cells. 2019;8(6).

35. Jin Q, Yan C, Qiu J, Zhang N, Lin Y, Cai Y. Structural characterization and deposition of stone cell lignin in Dangshan Su pear. Scientia Horticulturae .2013;155:123-130.

36. Cai Y, Li G, Nie J, Lin Y, Nie F, Zhang J, Xu Y. Study of the structure and biosynthetic pathway of lignin in stone cells of pear. Scientia Horticulturae .2010;125(3):374-379.

37. Dong X, Jiang Y, Hur Y. Genome-Wide Analysis of Glycoside Hydrolase Family 1 beta-glucosidase Genes in Brassica rapa and Their Potential Role in Pollen Development. Int J Mol Sci .2019;20(7). 
38. Cao Y, Han Y, Li D, Lin Y, Cai Y. MYB Transcription Factors in Chinese Pear (Pyrus bretschneideri Rehd.): Genome-Wide Identification, Classification, and Expression Profiling during Fruit Development. Front Plant Sci .2016;7:577.

39. Altenhoff AM, Dessimoz C. Inferring orthology and paralogy. Methods Mol Biol .2012;855:259-279.

40. Velasco R, Zharkikh A, Affourtit J, Dhingra A, Cestaro A, Kalyanaraman A, Fontana P, Bhatnagar SK, Troggio M, Pruss D, et al. The genome of the domesticated apple (Malus $x$ domestica Borkh.). Nat Genet .2010;42(10):833-839.

41. Higo K, Ugawa Y, Iwamoto M, Korenaga T. Plant cis-acting regulatory DNA elements (PLACE) database: 1999. Nucleic Acids Res. 1999;27(1):297-300.

42. Kristoffersen P, Brzobohaty B, Höhfeld I, Bako L, Melkonian M, Palme K. Developmental regulation of the maize Zm-p60.1 gene encoding a beta-glucosidase located to plastids. Planta. 2000;210(3):407415.

43. Biely P, Ahlgren JA, Leathers TD, Greene RV, Cotta MA. Aryl-glycosidase activities in germinating maize. Cereal Chem. 2003; 80:144-147.

44. Xie M, Huang Y, Zhang Y, et al. Transcriptome profiling of fruit development and maturation in Chinese white pear (Pyrus bretschneideri Rehd). BMC Genomics. 2013;14(1):823.

45. Opassiri R, Pomthong B, Onkoksoong T, Akiyama T, Esen A, Ketudat Cairns JR. Analysis of rice glycosyl hydrolase family 1 and expression of Os4bglu12 beta-glucosidase. BMC Plant Biol .2006;6:33.

46. Gomez-Anduro G, Ceniceros-Ojeda EA, Casados-Vazquez LE, Bencivenni C, Sierra-Beltran A, MurilloAmador B, Tiessen A: Genome-wide analysis of the beta-glucosidase gene family in maize (Zea mays L. var B73). Plant Mol Biol 2011, 77(1-2):159-183.

47. Cao Y P, Li D H, Meng D D, Jin Q, Cai Y P, Lin Y. Structural, Evolutionary, and Functional Analysis of the Class III Peroxidase Gene Family in Chinese Pear (Pyrus bretschneideri) [J]. Frontiers in Plant Science, 2016;7:1874.

48. Cannon SB, Mitra A, Baumgarten A, Young ND, May G. The roles of segmental and tandem gene duplication in the evolution of large gene families in Arabidopsis thaliana. BMC Plant Biol. 2004;4(1):10.

49. Cao Y, Han Y, Meng D, Li D, Jiao C, Jin Q, Lin Y, Cai Y: B-BOX genes: genome-wide identification, evolution and their contribution to pollen growth in pear (Pyrus bretschneideri Rehd.). BMC Plant Biol 2017, 17(1):156.

50. Punta M, Coggill PC, Eberhardt RY, Mistry J, Tate J, Boursnell C, Pang N, Forslund K, Ceric G, Clements J. The Pfam protein families database. Nucleic Acids Res. 2011;40(D1): D290-301.

51. Letunic I, Doerks T, Bork P. SMART 7: recent updates to the protein domain annotation resource. Nucleic Acids Res. 2012;40(D1): D302-5.

52. Kumar S, Stecher G, Tamura K. MEGA7: molecular evolutionary genetics analysis version 7.0 for bigger datasets. Mol Biol Evol. 2016;33(7):1870-4. 
53. Bailey TL, Johnson J, Grant CE, Noble WS. The MEME Suite. Nucleic Acids Research. 2015;43(W1): W39-W49.

54. Hu B, Jin J, Guo YA, Zhang H, Luo J, Gao G. GSDS 2.0: an upgraded gene feature visualization server. Bioinformatics. 2014;31(8):1296.

55. Wang Y, Tang H, DeBarry JD, Tan X, Li J, Wang X, Lee T-H, Jin H, Marler B, Guo H. MCScanX: a toolkit for detection and evolutionary analysis of gene synteny and collinearity. Nucleic Acids Res. 2012;40(7): e49.

56. Zhou H, Qi K, Xing L, Hao Y, Peng W, Chen J, Wu J, Zhang S. Genome-wide identification and comparative analysis of the cation proton antiporters family in pear and four other Rosaceae species. Mol Gen Genomics. 2016;291(4):1727.

57. Wu T, Zhang R, Gu C, Wu J, Wan H, Zhang S, Zhang S. Evaluation of candidate reference genes for real time quantitative PCR normalization in pear fruit. Afr J Agric Res. 2012; 7:3701-4.

58. Livak KJ, Schmittgen TD. Analysis of relative gene expression data using real-time quantitative PCR and the 2- $\Delta \Delta C T$ method. Methods. 2001;25(4):402-8.

\section{Figures}

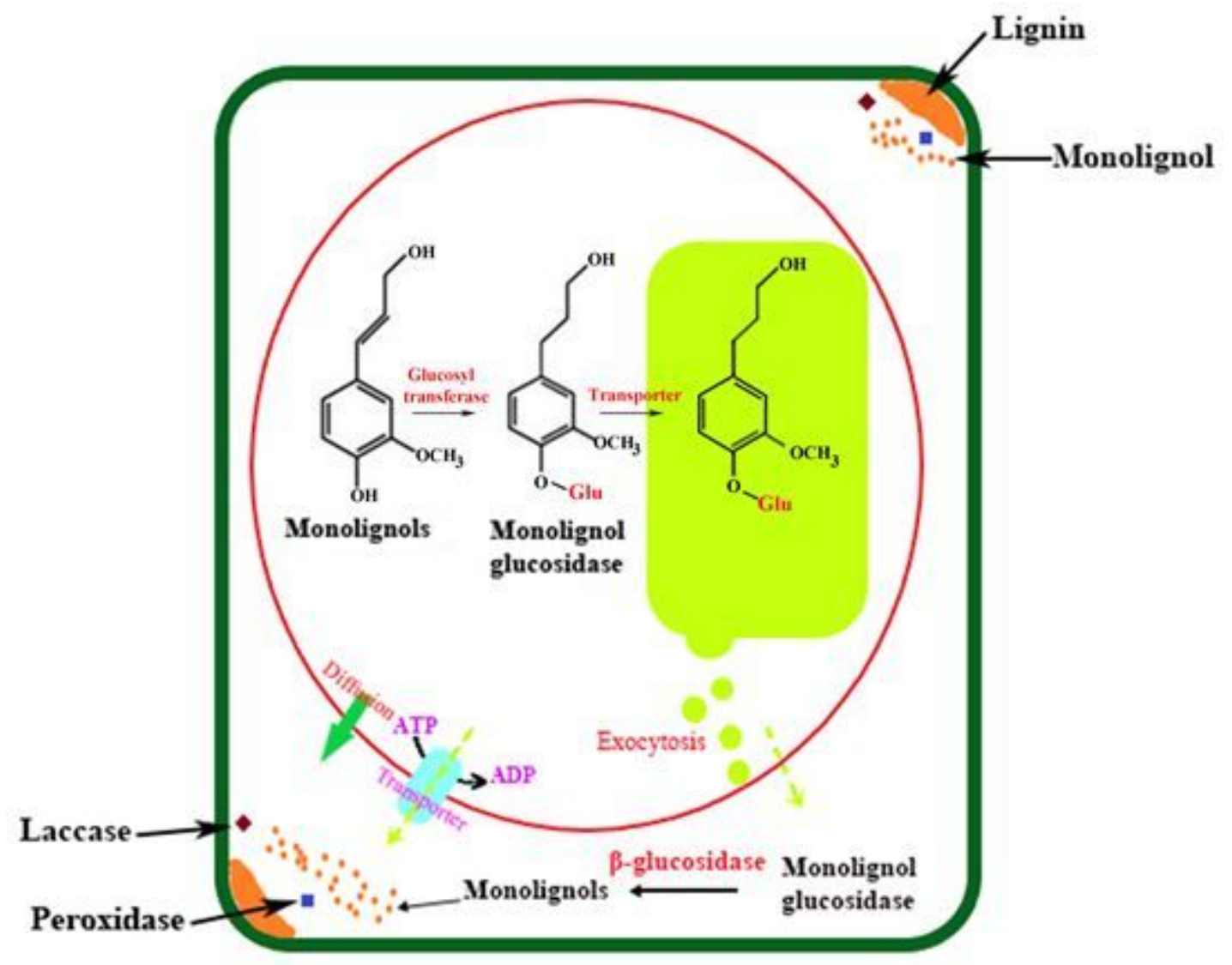


Figure 1

Mechanism of lignin monomer transport and deposition in cells

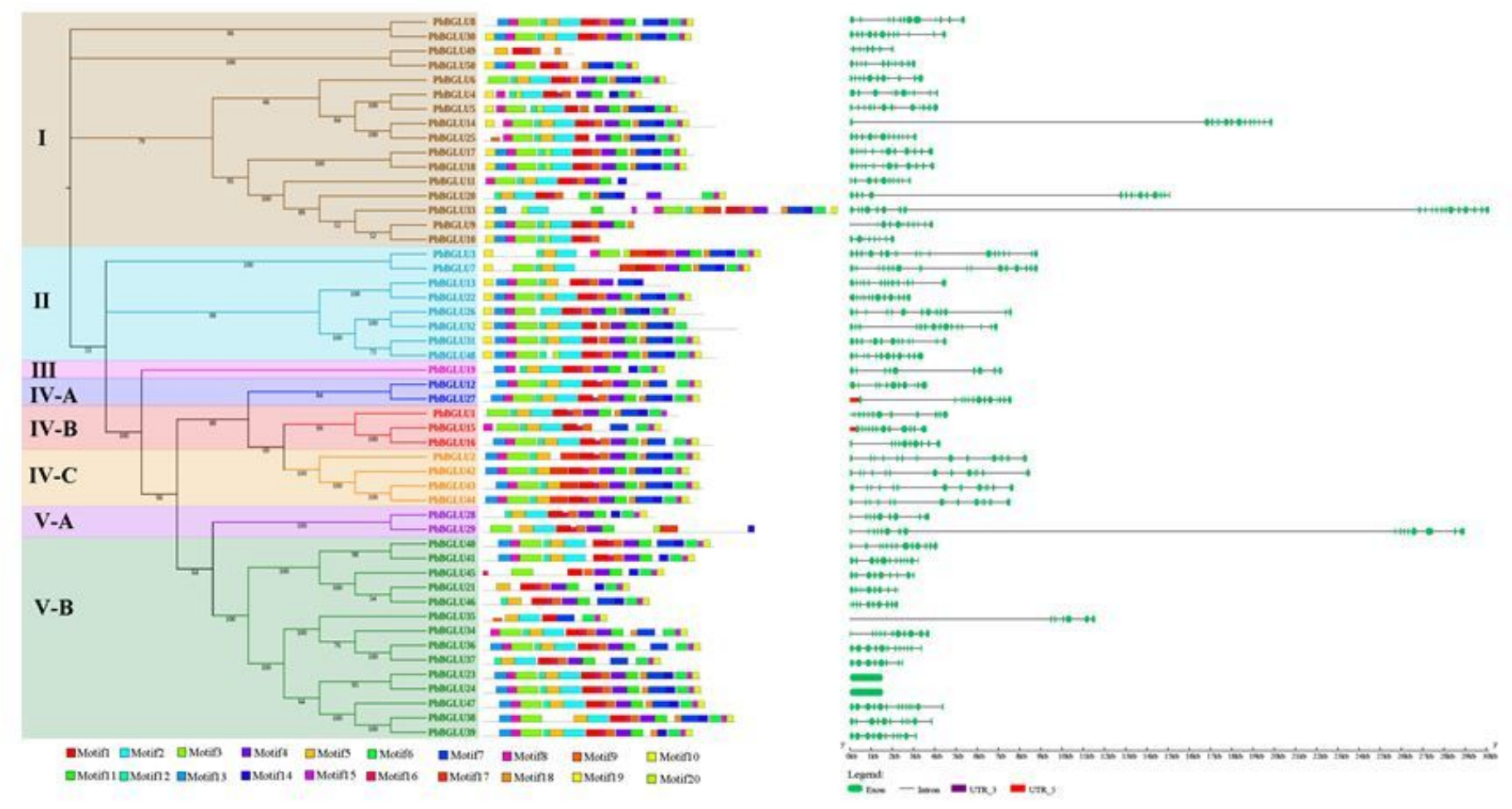

Figure 2

Phylogeny, conserved motifs and Exon-intron structure of BGLU genes in pear. MEME tools were used to identify motifs. Different colors represent different motifs. GSDS online website was used to generate structure. The legend is located at the bottom right of the figure. Scale Represents the length of the DNA sequence. 


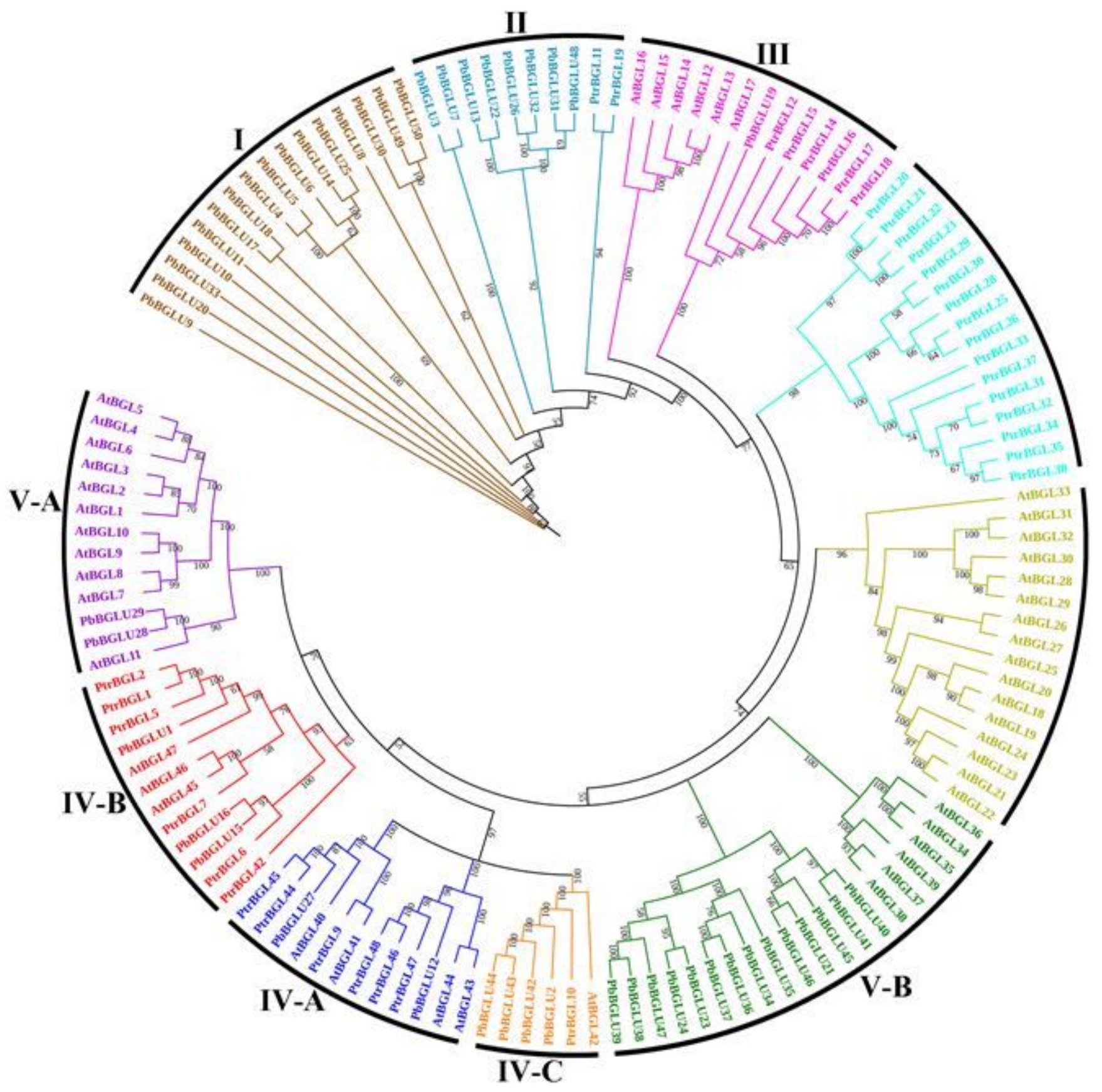

Figure 3

Phylogenetic analysis of BGLUs from pear, Arabidopsis and Poplar. The neighbor-joining (NJ) method was used to construct the phylogenetic tree 


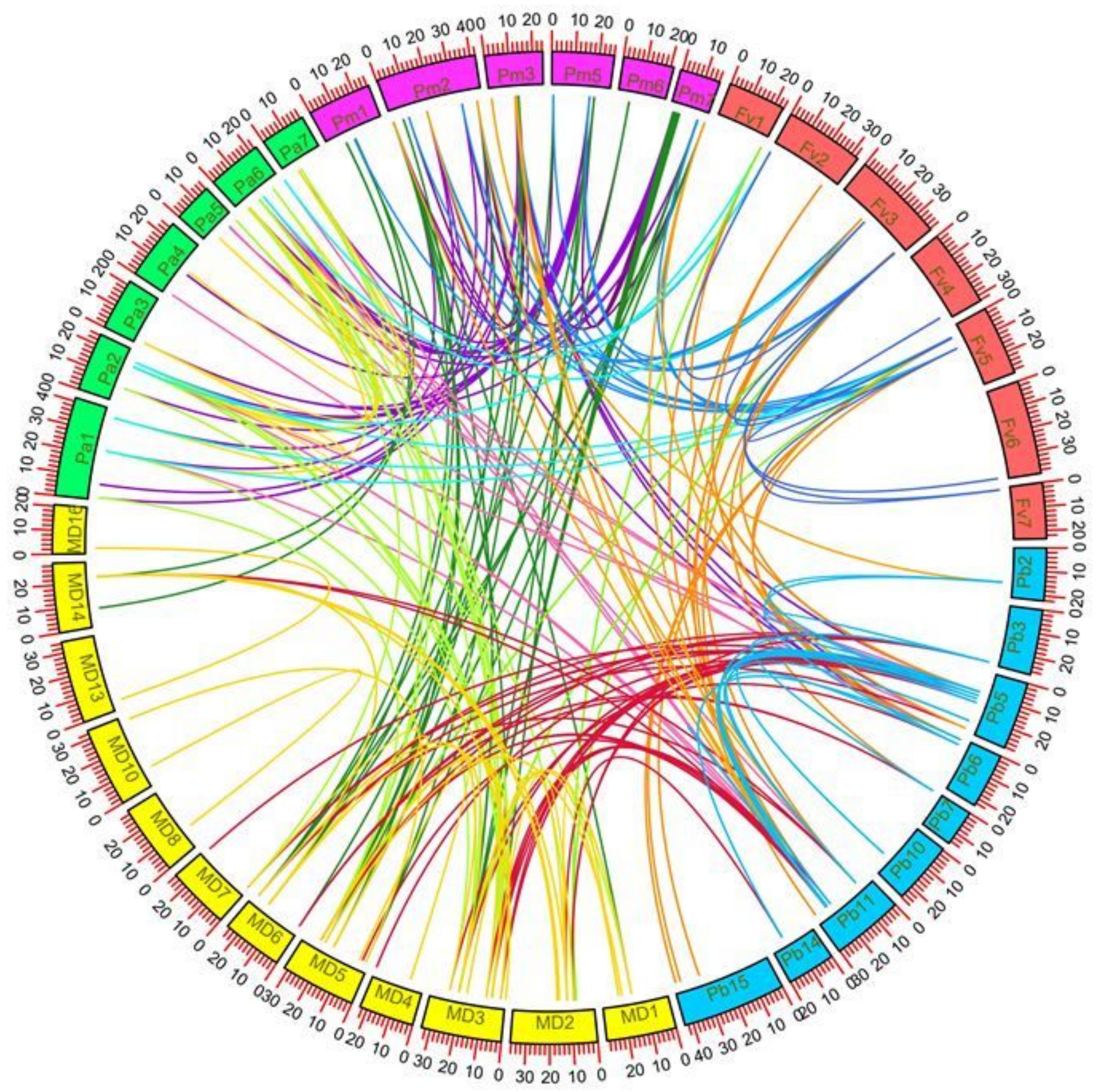

Figure 4

collinearity analysis of regions among Pyrus bretschneideri, Prunus mume, Malus domestica, Prunus avium, Fragaria vesca. The chromosome numbers are indicated by differently colored boxes and are labelled by $\mathrm{Pb}, \mathrm{Pm}, \mathrm{Md}, \mathrm{Pa}$ and $\mathrm{Fv}$. The differently colored boxes also represent the sequence lengths of chromosomes in megabases. The different color line indicates the syntenic relationship among the BGLU genes. 

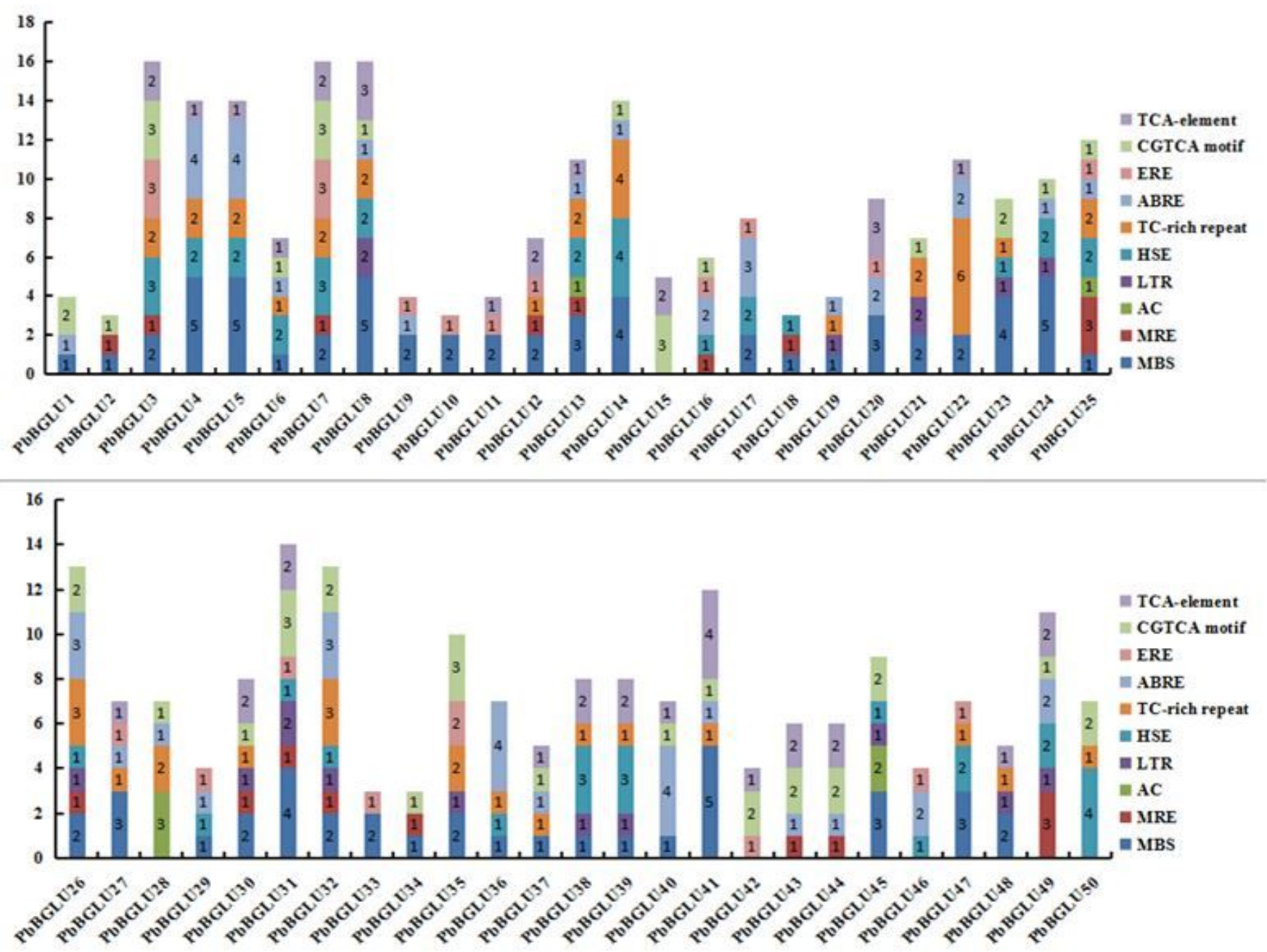

Figure 5

Promoter Cis-elements of the 50 PbBGLUs. Different colors represent Cis-elements. 

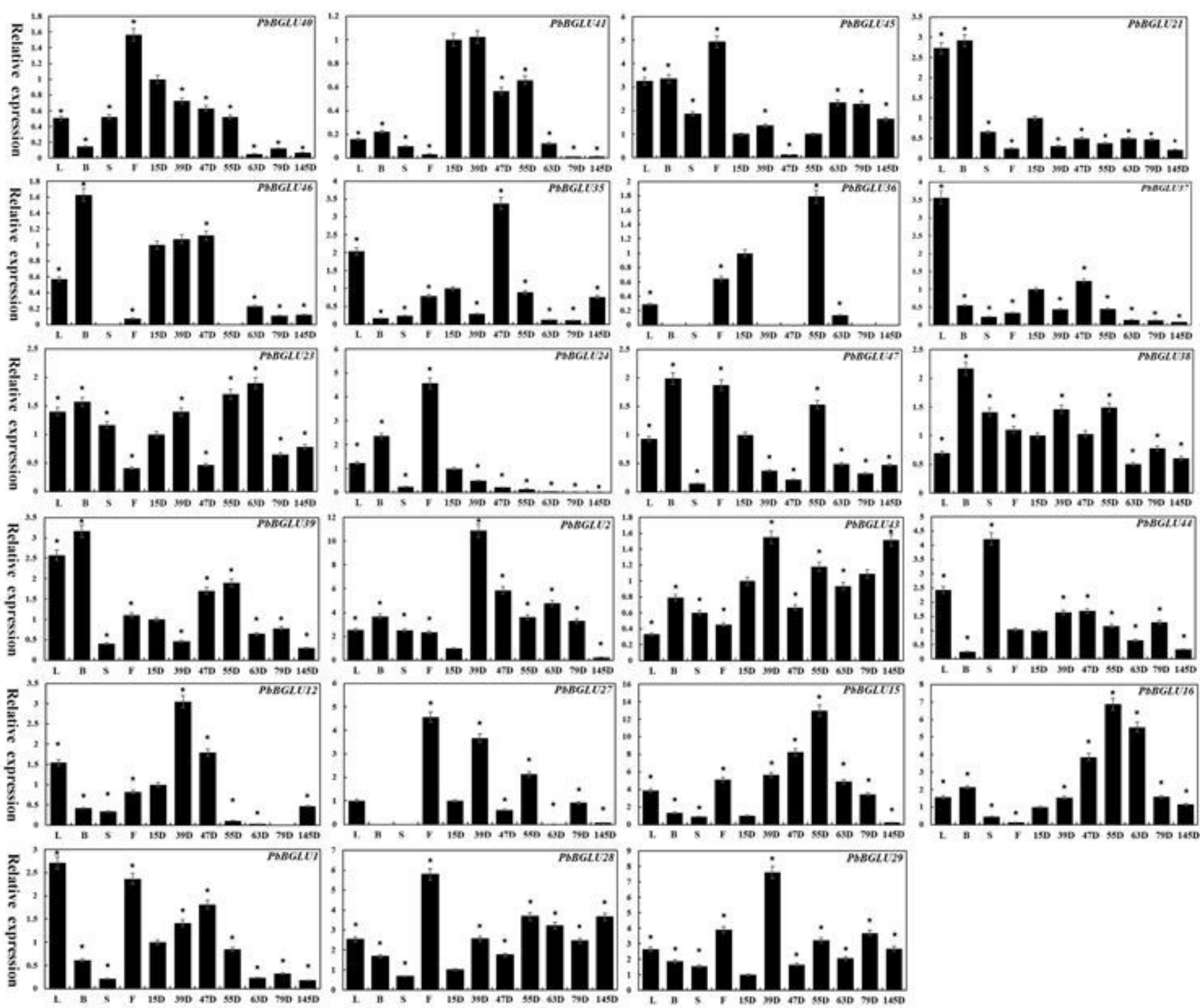

Figure 6

Expression levels of PbBGLU genes in different plant tissues. 15D, 39D, 55D, 79 D and $145 \mathrm{D}$ correspond to five different developmental stages (days after flowering) of pear fruit. In addition, leaf, bud, stem and flower were represented by $L, B, S$ and $F$, respectively. The value on the left $Y$-axis indicates the relative gene expression levels. * significant difference at $\mathrm{P}<0.05$. 


\section{Fluorograph Maciograph Merge}
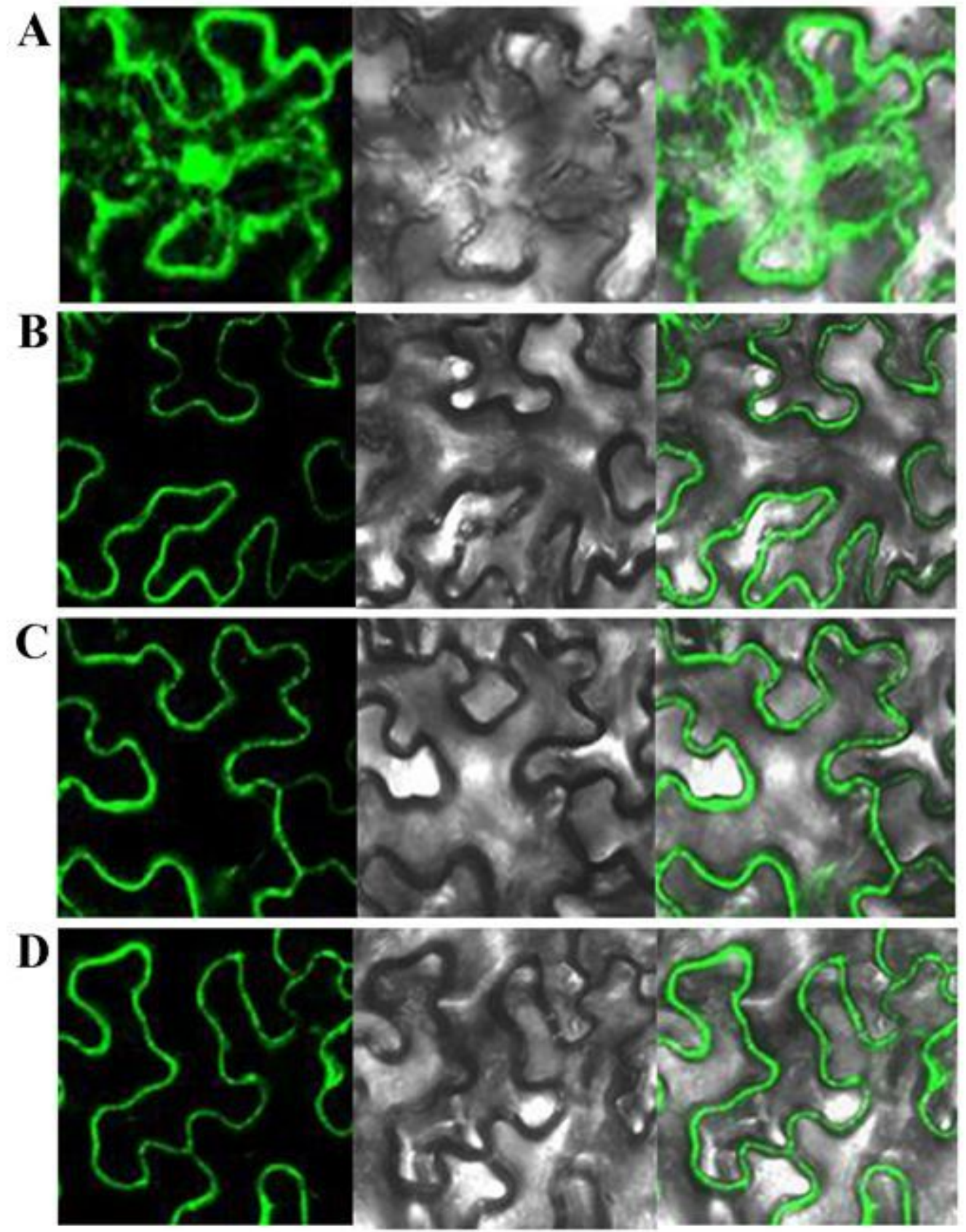

Figure 7

Subcellular localization of PbBGLU1-GFP, PbBGLU15-GFP and PbBGLU16-GFP fusion protein. (A) GFP control. (B) Subcellular localization of PbBGLU1-GFP fusion protein. (C) Subcellular localization of PbBGLU15-GFP fusion protein. (D) Subcellular localization of PbBGLU16-GFP fusion protein.

\section{Supplementary Files}


This is a list of supplementary files associated with this preprint. Click to download.

- Additionalfile1.xlsx 